\title{
Trunk Movements During Locomotion in the Marsupial Monodelphis domestica (Didelphidae)
}

\author{
PETER A. PRIDMORE \\ Division of Biological Sciences, University of Michigan, Ann Arbor, \\ Michigan 48109
}

\begin{abstract}
The small didelphid marsupial, Monodelphis domestica, uses a lateral sequence walk during slow treadmill locomotion and gradually shifts to a trot as speed increases. At higher speeds it changes abruptly to a half-bound. Cinematographic records suggest significant lateral bending but no sagittal bending of the trunk during the slow walk and a reduced amount of lateral bending during the fast walk. There is slight lateral, but no sagittal, bending during the trot. Sagittal bending is obvious during the half-bound, but no lateral bending is evident. Cineradiography confirms that the vertebral column of the trunk bends laterally during the slow walk. Bending occurs throughout the trunk region, but seems to be most pronounced in the anterior lumbar region. Associated with this bending of the trunk is substantial rotation of the pelvic girdle in the plane of yaw. Pelvic rotation is synchronized with the locomotor cycle of hindlimbs. Each side of the pelvis rotates forward during the recovery phase of the ipsilateral hindlimb and backward during the contact phase of this limb. Information on locomotor trunk movements in other limbed tetrapods is limited. The pattern of trunk bending found in Monodelphis, however, is consistent with that reported in the placental mammal Felis catus and in some lepidosaurian reptiles. This suggests that sagittal bending did not replace lateral bending during the evolution of mammals, as is sometimes suggested. Rather, bending in the vertical plane seems to have been added to lateral bleeding when the ancestors of extant mammals acquired galloping and bounding capabilities.
\end{abstract}

Sagittal bending of the trunk characterizes high speed locomotion in many mammals (Slijper, '46; Gambaryan, '74). In contrast, locomotion in limbed reptiles and amphibians is usually associated with lateral bending of the trunk (Daan and Belterman, '68; Roos, '64; Sukhanov, '74). In his 1944 book Speed in Animals, A. Brazier Howell discussed movements of the axial skeleton in terrestrial vertebrates. He suggested that lateral bending is associated with symmetrical gaits (walking, trotting) and sagittal bending with gallop-type gaits. This correlation of bending plane with gait implies that lateral bending ought to be apparent in most mammals moving at slow to moderate speeds. However, since Howell's time little consideration has been given to the matter.

Jenkins ('71) and Jenkins and Camazine ('77) in studies of mammalian hindlimb function demonstrated considerable horizontal rotation of the pelvis (rotation in the plane of yaw) in the Virginia opossum and in several species of carnivore and noted in passing that minimizing lateral displacements of the trunk might be important for predators while stalking prey. However, direct investigation of lateral bending in the mammalian trunk has been limited to studies on a single species: Carlson et al. ('79) demonstrated small lateral flexions of the posterior trunk of cats during movement at low speed and provided evidence that the extent of such bending varies with locomotor speed.

This raises the question whether lateral bending is a common feature of slow locomotion in mammals and also whether such bending varies with speed in a consistent manner in various mammalian types. These issues are important because the presence of lateral

Peter Pridmore is now at the Department of Geology, Australian National University, Canberra, A.C.T. 2601, Australia. Address reprint requests there. 
bending has major implications for the structural and functional changes that occurred during the descent of mammals from their reptilian ancestors.

The present study was undertaken to describe the axial movements found at different speeds in a mammal very distantly related to the domestic cat. Direct observations and preliminary cinematographic records suggested that considerable lateral bending of the trunk occurs during low speed locomotion in the small didelphid Monodelphis domestica. Locomotion over a range of speeds was, therefore, characterized in this species using cinematography. Following this, cineradiographic methods were used to show the orientations assumed by the spinal column of live specimens of $M$. domestica during locomotion and to evaluate the extent to which bending of the trunk vertebrae can be recognized from cinematographic records. Part of this study has been reported previously (Pridmore, '83).

\section{MATERIALS AND METHODS}

Cinematographic studies were carried out using 4 adult $M$. domestica (80 to $125 \mathrm{~g}$ in body weight), which had been trained to run on a treadmill within a plexiglass box $(43 \mathrm{~cm}$ long, $13 \mathrm{~cm}$ wide, $12 \mathrm{~cm}$ high). A mirror mounted at $45^{\circ}$ above the box permitted simultaneous lateral and dorsal views. Film records were obtained using a Canon Autozoom 814 Electronic Super-8 movie camera run at 40 frames per second.

Film sequences showing steady locomotion were identified and subjected to frame-byframe analysis to determine gait (Hildebrand, '66, '76) and locomotor speed. Selected frames were then projected onto white drawing paper, and the outline of the animal in both lateral and dorsal views traced to document changes in whole body outline and limb position throughout the locomotor cycle.

Cineradiographic studies used 2 adult animals (male $122 \mathrm{~g}$; female $85 \mathrm{~g}$ ). These animals were induced to locomote along $91.5 \mathrm{~cm}$ long pieces of wood of various width sthrough a ventro-dorsal radiographic field. A flat substrate was provided by a length of wood $9 \mathrm{~cm}$ wide and $1.3 \mathrm{~cm}$ thick. This was fitted with small tacks at $6 \mathrm{~cm}$ intervals to serve as markers. Lengths of dowel of $6.3 \mathrm{~mm}, 19$ $\mathrm{mm}$, and $39 \mathrm{~mm}$ diameter served as rounded substrates. The thicker dowels were fitted with tacks, but the $6.3 \mathrm{~mm}$ dowel was too thin to carry them. However, by maintaining substrate position with respect to the X-ray unit, it was possible to scale radiographs of locomotion on the narrow dowel by comparing them with records of progress along the other substrates. A Philips Diagnost 'C' Type XG 5002 Cinefluorographic X-ray unit provided the radiographic field. The cinefluoroscope of the X-ray unit was connected either to a Panasonic VHS portable cassette recorder or to a $70 \mathrm{~mm}$ spot film device to obtain radiographic records. Video records were collected at 60 frames per second. Spot filming was carried out at 6 frames per second with a $50 \mathrm{msec}$ exposure time. X-ray factors of $65 \mathrm{kV}$ and $950 \mathrm{Ma}$ were used throughout.

\section{RESULTS \\ Cinematography}

At low speeds Monodelphis uses a lateral sequence walk (sensu Hildebrand, '66, '76). If the set-down of the left hindfoot is taken as the start of the locomotor cycle, the remaining feet are set down in the order left front, right hind, right front (Fig. 1A). With increasing speed, animals move progressively through faster forms of the lateral sequence walk into a trot (Fig. 1D) in which the diagonal limb pairs function in synchrony. The phase relations of limbs on opposite sides of a limb girdle do not alter during this progression; however, the phase relations between the pectoral and pelvic limb girdles undergo a shift. Thus, the forefoot during the slow walk follows its ipsilateral hindfoot by approximately $30 \%$ of the duration of the locomotor cycle (Fig. 1A), but lags it by approximately $50 \%$ in the trot (Fig. 1A).

A reduction in the absolute duration of the locomotor cycle (stride interval) is also associated with the progression from lateral sequence walk to trot. This reduction is great when the animal increases speed from a slow walk, but at a faster walk the reduction for a given speed increment is relatively less. The locomotor cycle of any limb involves a contract or stance phase and a non-contact or swing phase. The reduction in stride interval observed in the walk-trot progression is primarily the result of a reduction in the duration of the contact (or stance) phase. The duration of the non-contact (swing) phase decreases only slightly as speed is increased.

The abrupt transition from the trot to the half-bound contrasts sharply with the gradual nature of that between walk and trot. The transition to the half-bound involves a sudden change in the phase relations of limbs 
A

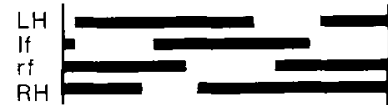

0.25

$\mathrm{m} / \mathrm{sec}$

B

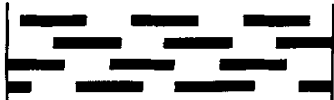

0.57

$\mathrm{m} / \mathrm{sec}$

C

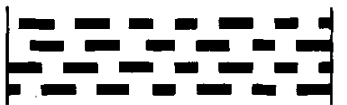

1.11

$\mathrm{m} / \mathrm{sec}$

D

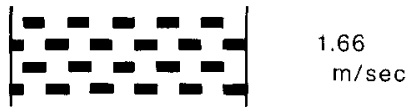

E
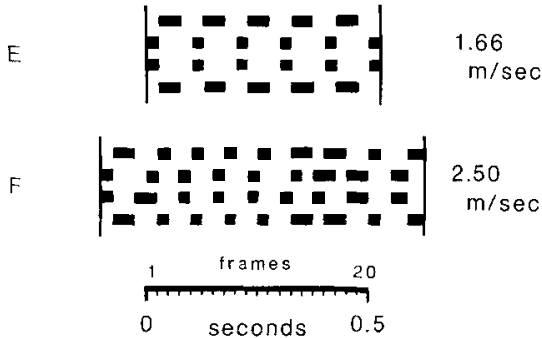

Fig. 1. Monodelphis domestica. Gait diagrams for travel at various speeds. Time runs from left to right in each diagram and the common time scale is indicated at the bottom of the figure. Solid bars indicate contact (stance) phases for each limb. During a slow lateral sequence walk (A) the set-down of the left hindlimb (LH) is preceeded by the set-down of the right forelimb (rf) about $0.1 \mathrm{sec}$ earlier and followed by set-down of the left forelimb (If) about 0.15 sec later. In successively faster lateral sequence walks $(\mathbf{B}, \mathbf{C})$ the cycle duration is reduced and the phase relations are modified so that the diagonal limbs increasingly converge in phase. During a trot (D) the cycle duration is further reduced and the diagonal limbs act in complete synchrony so that the animal is alternatively supported by one pair of diagonal limbs and then the other. In a half-bound (E, F) quite different patterns of limb activation are used; the two hindlimbs act in synchrony and the left and right forelimbs almost so.

on opposite sides of the limb girdles. The half-cycle phase difference initially existing across both girdles (during trot and walk) is suddenly reaplced by in-phase activity of the two hindlimbs and near in-phase activity of the two forelimbs (Fig. 1E,F).

The speed at which the walk-trot transition occurs cannot be so readily determined because the transition between these two gaits involves no obvious modification of the pattern of movement of either the limbs or trunk. That the transition ha: veen made can only be recognized by the occurrence of free-flight phases in the locomotor cycle, and such free-flight phases are extremely brief at speeds immediately above the transition. The dramatic modification of phase relations of the limbs makes the trot-half-bound transition more obvious than the walk-trot transition, as does the advent of substantial sagittal bending of the trunk at speeds beyond the transition.

At speeds above the walk-trot transition, both the locomotor cycle and its contact phase have a more-or-less constant duration suggesting that animals increase their velocity while trotting or half-bounding by extending stride length.

Lateral views of walking (Fig. 2) and trotting (Fig. 3) animals suggest that sagittal bending is at most slight during these gaits. However, during the half-bound the trunk shows marked flexion and extension in the sagittal plane (Fig. 4).

Dorsal views of locomotion indicate that the trunk undergoes significant lateral bending during a slow walk (Fig. 5A). In a faster walk (Fig. 5B), the magnitude of lateral blending is reduced. At both speeds, one complete bending cycle occurs with each locomotor

\section{$0.19 \mathrm{~m} / \mathrm{sec}$}
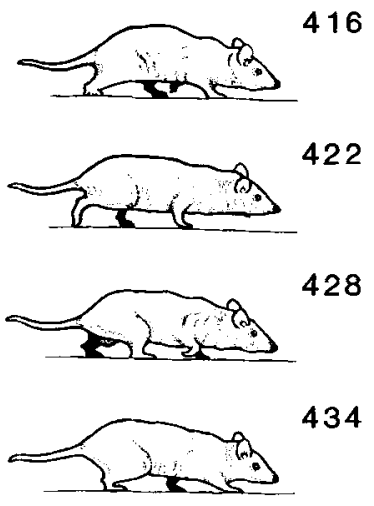

434

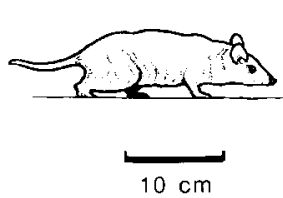

440

Fig. 2. Monodelphis domestica. Slow lateral sequence walk. In frame 416 placement of the left hind foot has just occured and the left front foot has just been lifted. The left front foot is then set down (between frames 422 and 428), followed by the right hind foot (immediately prior to frame 428) and the right front foot (between frames 428 and 434 ). 


\section{$1.66 \mathrm{~m} / \mathrm{sec}$}

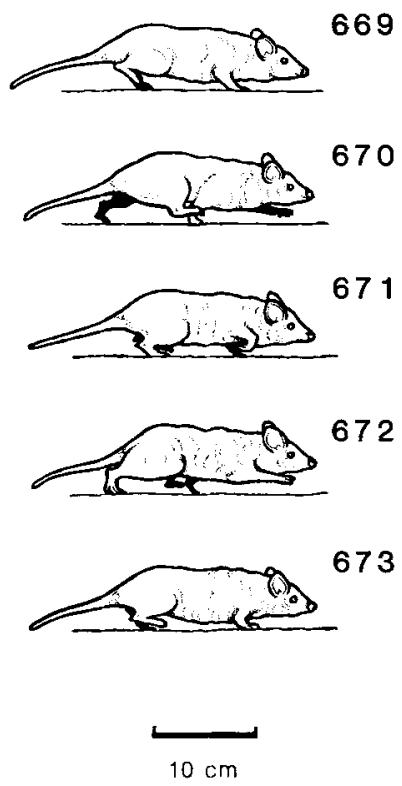

Fig. 3. Monodelphis domestica. Trot. In frames 669 and 670 the animal is supported by its right front and left rear limbs. In frames 671 and 672 the supporting limbs are those of the left front and right rear. Note that there is no indication of any bending of the trunk in the sagittal plane in this figure or in Figure 2.

cycle. Regardless of walking speed, the trunk shows maximum bending at the stage in the locomotor cycle in which the hindfoot on the concave side of the trunk is being set down. Dorsal views of animals trotting show very little lateral bending (Fig. 6A). There is no evidence of lateral bending during half-bounding (Fig. 6B).

\section{Cineradiography}

Cineradiographic records reveal lateral bending of the trunk vertebrae during walking on both round and flat substances. Bending is most pronounced in radiographs obtained using the $6.3 \mathrm{~mm}$ dowel as a substrate (Fig. 7), All locomotion along this substrate was slow, which made it suitable for investigation with the low framing rate of the spot film device. Moreover, as indicated earlier, lateral bending is most pronounced in Monodelphis during slow walking.

Figure 7 and other radiographic records show that bending takes place throughout the trunk. The extent of lateral bending appears to be greatest in the anterior lumbar

\section{$2.35 \mathrm{~m} / \mathrm{sec}$}

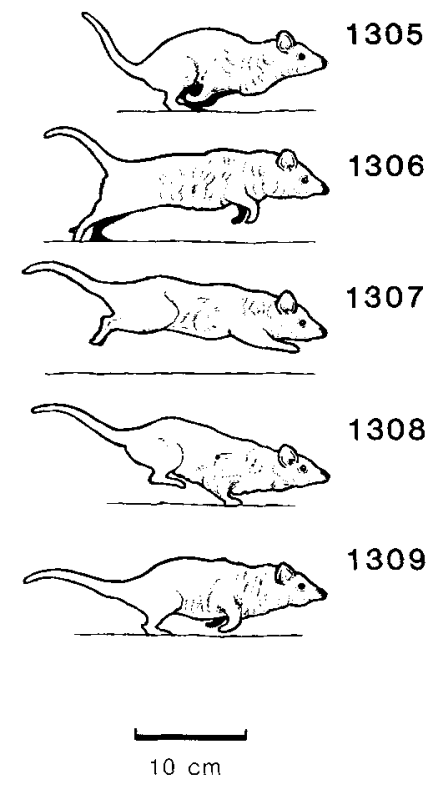

Fig. 4. Monodelphis domestica. Half-bound. In frame 1305 the animal has just lifted its forelimbs prior to a period of extended flight (shown in progress in frame 1307). This period of extended flight concluded immediately prior to frame 1308 . Frame 1309 shows the animal in a stage preceeding a second period of extended flight. The stage shown in frame $\mathbf{1 3 0 9}$ is actually a little more advanced in the locomotor cycle than that shown in frame 1305. Note the pronounced bending of the animal's trunk in the sagittal plane in this mode of locomotion. Compare particularly the extreme flexion of the trunk shown in frame 1305 with the extension shown in frame 1306 .

region, but other regions contribute significantly to the arc formed by the spinal column when it is maximally bent. Furthermore, the anterior thoracic vertebrae bend in the opposite direction to those more posteriorly situated, with the result that the neck and head are directed forward rather than to the side (Fig. 7).

Radiographic records also revealed that substantial horizontal rotation of the pelvic girdle occurs during trunk bending. In Figure 7 the pelvic makes a total rotation of $30^{\circ}$ to $40^{\circ}$ within the plane of yaw in the course of a single locomotor cycle.

On other substrates, locomotion was faster with the result that radiographic records present a less complete picture of the orientations assumed by the vertebral column within a single locomotor cycle. Figures 8-10 show radiographs obtained during traverses of the 

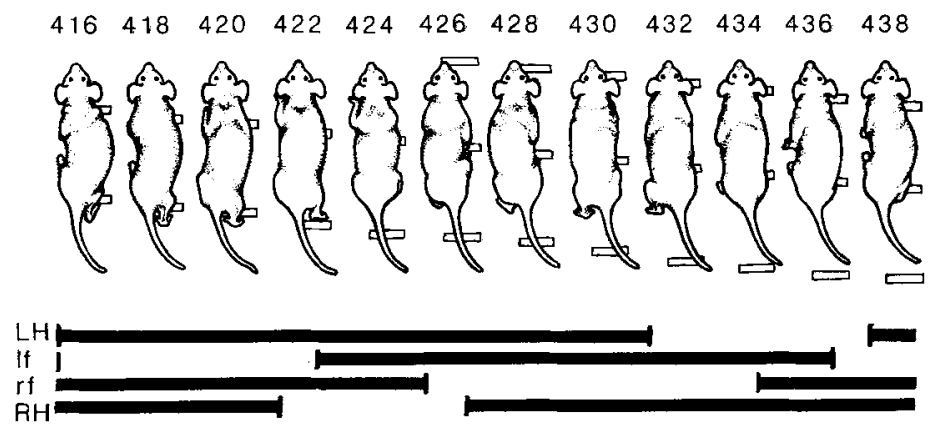

(B)

Speed: $0.57 \mathrm{~m} / \mathrm{sec}$

(3.5 Body L/sec)
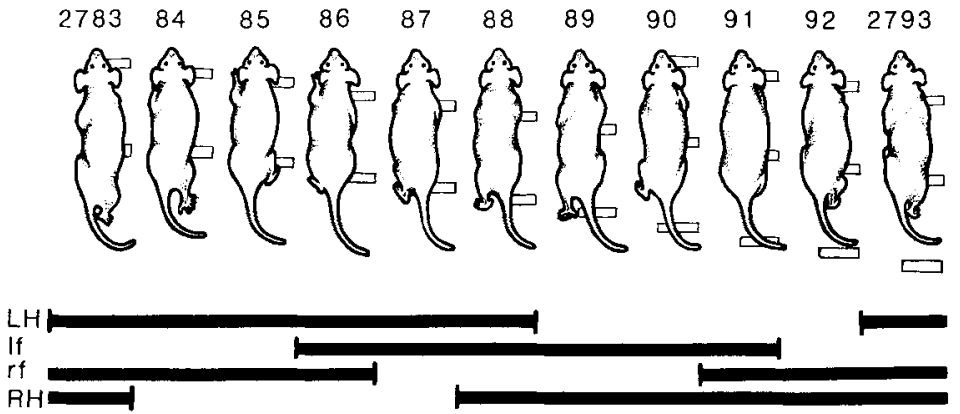

Fig. 5. Monodelphis domestica. Dorsal views of a lateral sequence walk. The sequence shown in $\mathbf{A}$ is for a slow walk, that in $\mathbf{B}$ for a faster one. The earliest stage in each sequence is on the extreme left and successive stages follow to the right. Gait diagrams are shown

$9 \mathrm{~cm}$ wide board. Despite the small number of records obtained each time the animal passed through the radiographic field, it is clear that lateral bending of the vertebral column occurs during locomotion on this flat substrate and that such bending is not an artifact occasioned by the use of a round locomotor surface. In addition, these records give clear evidence of rotation of the pelvis in the plane of yaw and indicate substantial lateral bending in the anterior lumbar region.

\section{DISCUSSION}

The gait repertoire of Monodelphis domestica is similar to that of the many small to beneath each series of drawings. Note the more pronounced lateral curvature of the trunk at the slower walking speed (A). At both speeds truncal curvature is greatest at or soon after the setdown of a hindfoot.

moderate sized therians (e.g., Gambaryan, '74; Goslow, et al., '73) that use a lateral sequence walk as their slowest gait. At higher speeds these animals trot and at the highest speeds they half-bound or bound. The lateral sequence walk and the trot are also used by limbed lepidosaurian reptiles and urodele amphibians (Rooos, '64; Dann and Belterman, '68; Sukhanov, '74); however, these latter forms evidently lack the half-bound and bound.

Lateral bending has been reported in a variety of limbed reptiles and urodele amphibians, but few studies have considered how the extent of bending varies with speed. $\mathrm{Cu}$ riously, studies that do consider the latter 
(A)

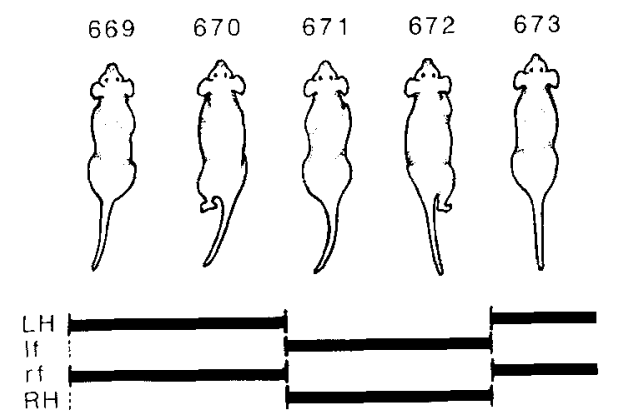

(B)

Speed: $2.35 \mathrm{~m} / \mathrm{sec}$ (14.7 Body L/sec)
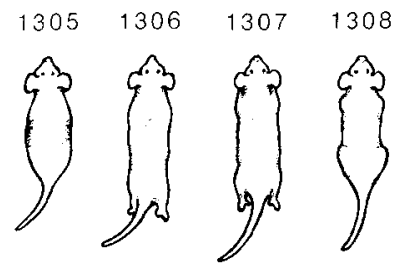

\section{9}
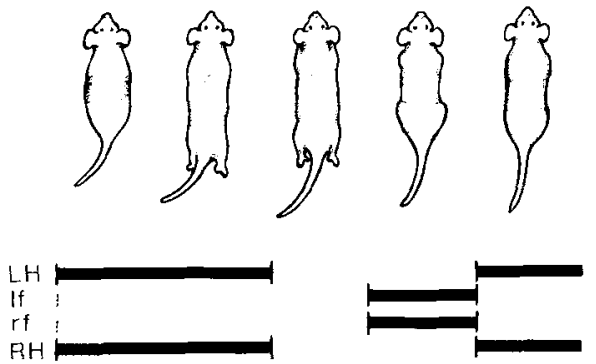

Fig. 6. Monodelphis domestica. Dorsal views of trot (A) and half-bound (B). The first stage in each sequence is the extreme left and successive stages follow to the right. Lateral curvature of the trunk is slight during the trot and not evident at all during the half-bound.

issue indicate contradictory findings. Edwards ('76) implied that the amount of lateral bending in terrestrial salamanders increases with speed, but presented no relevant data. Snyder ('62) and Sukhanov ('74) claimed that lizards increase lateral bending with speed, but neither presented material to demonstrate this. Opposite claims have been made for some lizards by other workers. Lateral bending has been most thoroughly investigated in Lacerta viridis, and according to Daan and Belterman ('68), the amount of bending falls off with speed in this species. This seems also to be the case with Varanus komodoensis (Auffenberg, '81), which is said to exhibit substantial lateral bending while walking but none while trotting. The amount of lateral bending recorded by Carlson et al.
('79) in walking cats was small, but also decreased with increasing speed. Thus the observation in Monodelphis that lateral bending decreases with speed is consistent with reports for some other quadrupedal amniotes.

Although the amount of lateral bending in both Monodelphis and Felis catus is greatest during slow walking, the small marsupial seems to show relatively more bending than the placental at such speeds. This may relate to the use of less erect limb orientations by Monodelphis. With regard to this, it is interesting that Jenkins and Camazine ('77) found considerably greater rotation in the plane of yaw of the pelves of racoons walking at 3.2 $\mathrm{km} / \mathrm{hr}$ than in those of more erect-limbed carnivores (a fox and cats) walking at the same speed. In these three carnivores, the amount of horizontal rotation of the pelvis during walking correlates with the extent to which the femur is abducted.

Aside from confirming the bending pattern indicated by cinematography, radiographic records are important for the information they provide on the influence of joint morphology on trunk bending. Although the trunk vertebrae of mammals are usually divided into thoracic and lumbar groups according to whether ribs are present or absent, the trunk can also be divided according to the orientation of zygapophyseal facets into preand post-diaphragmatic regions. In the anterior trunk (pre-diaphragmatic region) the vertebrae possess zygapophyseal facets that lie in a more-or-less horizontal plane. In the posterior of the trunk (post-diaphragmatic region) the equivalent facets are more upright and lie in an approximately parasagittal plane. In many mammals the transition between these two orientations of the facets takes place across one vertebra, the diaphragmatic, which bears horizontal prezygapophyseal and vertical postzygapophyseal facets (Slijper, '46). Several authors (Rockwell et al., '38; Washburn and Buettner-Janusch, '52) have pointed out that the orientation of the facets in the anterior trunk is permissive of lateral bending and restrictive of sagittal bending, whereas facetal orientation in the posterior trunk is conducive of sagittal bending but restrictive of both lateral bending and long-axis rotation.

Monodelphis domestica normally has 14 thoracic and 6 lumbar vertebrae (Pridmore, unpub. data). The diaphragmatic vertebra is usually the 10th or 11th thoracic vertebra, 

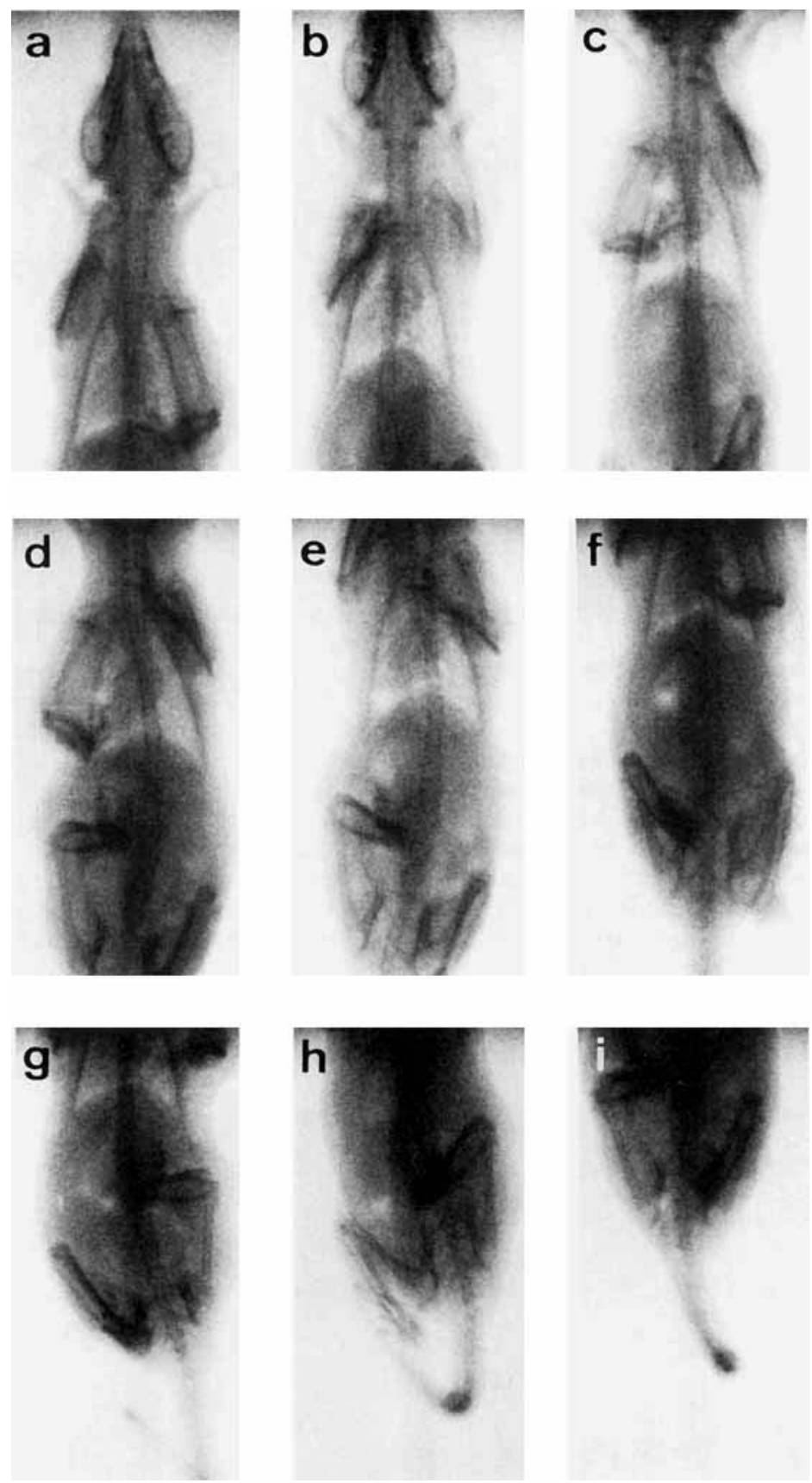

Fig. 7. Monodelphis domestica. Successive radiographic records of locomotion along $6.3 \mathrm{~mm}$ dowel. Locomotor speed is approximately $0.06 \mathrm{~m} / \mathrm{sec}$ and the total time elapsed over the entire 9 plates is $1.5 \mathrm{sec}$. In $\mathbf{d , e}$ and i the animal's spine is convex on the right and the anterior of its pelvis is directed forward and somewhat to the right. In $\mathbf{b}$ and $\mathbf{g}$ the opposite spinal curvature is evident and the pelvis is directed forward and a little to the left. 


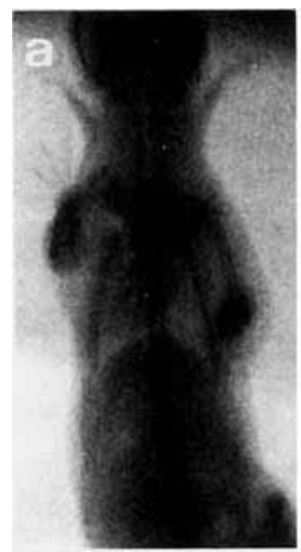

Fig. 8. Monodelphis domestica. Radiographic records of locomotion along $9 \mathrm{~cm}$ wide flat board. Successive records for an animal traveling at approximately 0.3

although in a few animals the zygagpophyseal facets interconnecting Th 10 and Th 11 show an intermediate condition. In individuals of the latter type a single diaphragmatic vertebra cannot be recognized; rather, there exists a "diaphragmatic region" consisting of Th 10 and Th 11. If zygapophyseal facetation influences trunk bending, different amounts or patterns of bending should be exhibited by intervertebral joints anterior and posterior to the 10 th or 11 th thoracic vertebra. The vertebral orientations observed with cineradiography in this study indicate that the amount of bending that occurs in a particular region of the trunk during locomotion cannot $\mathrm{m} / \mathrm{sec}$. In a the animal's spine is convex on the left whereas in $\mathbf{b}$ there is evidence of a slight convexity on the right.

be related simply to zygapophyseal facetation.

In reptiles and urodele amphibians, lateral bending has usually been interpreted as a mechanism for increasing stride length (e.g., Edwards, '76; Sukhanov, '74; Daan and Belterman, '68). According to this view, active bending of the trunk serves to move an advancing limb further forward during the recovery phase of the limb cycle. However, in Lacerta viridis stride length increases with speed, whereas maximal trunk curvature decreases (Daan and Belterman; '68). This does not show that bending makes no contribution to stride length, but it does call into

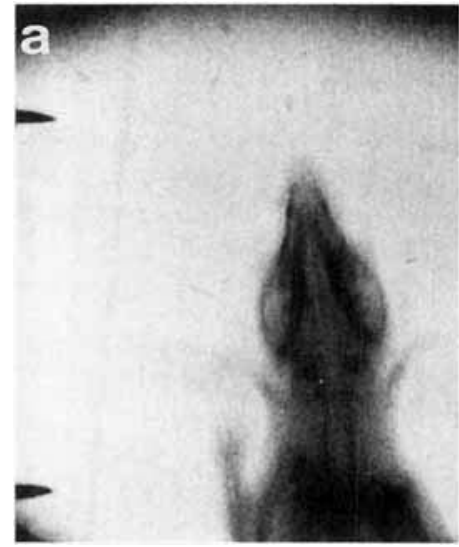

Fig. 9. Monodelphis domestica. Two successive records for the same animal as in Figure 8 progressing at approximately $0.4 \mathrm{~m} / \mathrm{sec}$. The dark marks to the sides of

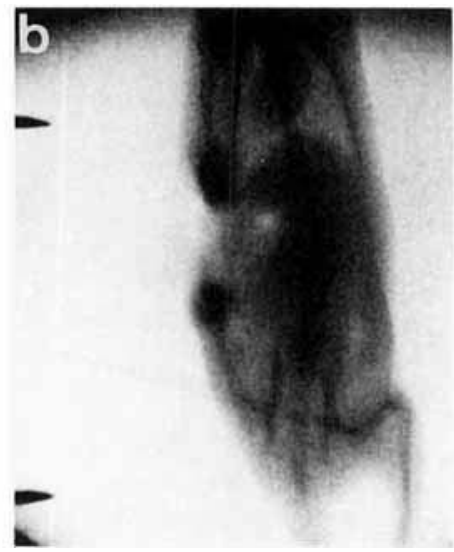

the animal are tacks set at $6 \mathrm{~cm}$ intervals along the edge of the substrate. Note the convexity on the right of the spine in (b). 

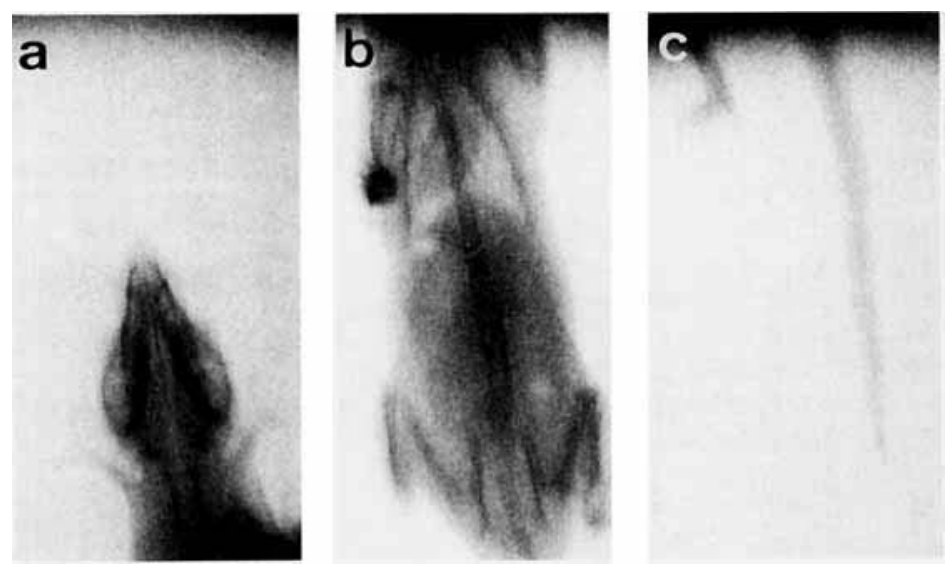

Fig. 10. Monodelphis domestica. Three successive records of the same animal as in Figures 8 and 9 traveling at $0.5 \mathrm{~m} / \mathrm{sec}$.

question the usual interpretation. Most tetrapods increase speed in the upper part of their locomotor range by substantially increasing stride length. It seems odd therefore, that a mechanism capable of doing this should become less operational with increasing speed.

In Felis and Monodelphis, lateral bending also decreases with increasing speed, and any substantial contribution of this to stride length can only be made when these animals are walking slowly. Interestingly, in humans pelvic rotation is increased with speed (Levens et al., '48; Inman et al., '81).

It has been assumed that lateral flexions of the trunk in limbed reptiles are actively brought about by axial muscles, but no evidence for this has ever been presented. Carlson et al. ('79) envision a quite different role for such muscles in Felis. They postulate that the primary role of the back muscles of this mammal during walking may be in controlling the stiffening of the back, rather than inducing movements. They suggest that lateral trunk movements may be induced by limb action. This implies that lateral bending of the trunk may be a consequence of pelvic rotation, rather than a cause of it.

In view of the similarities of the pattern of back movements in Felis, Monodelphis, and Lacerta, it is not inconceivable that the same factors are responsible for the lateral bending in all three animals, though whether limb muscles, trunk muscles, or both are involved remains to be determined.

The results obtained in the present study and those reported by Carlson et al. (79) are not without evolutionary interest. It has gen- erally been assumed that lateral bending of the trunk was characteristic of early reptiles and most of their descendants, including the early mammal-like reptiles. Kemp ('82) has suggested that lateral bending was lost within the latter lineage prior to attainment of the mammalian level of organization and that it was replaced with sagittal bending in mammals or their immediate ancestors. However, the observation that lateral bending occurs in both Monodelphis and Felis argues that neither mammals nor their ancestors ever lost lateral bending movements. Instead, it seems probable that sagittal bending movements were added to the lateral bending ones, presumably in conjunction with the acquisition of asymmetrical gaits (galloping and bounding). A similar sequence of changes seem to have taken place also within archosaurian reptiles. Both lateral and sagittal bending movements are observed in crocodilians. As in mammals, the former are associated with slow walking, while the latter only appear during galloping (Webb and Gans, '82; Zug, '74).

\section{ACKNOWLEDGMENTS}

Preliminary cinematographic records were obtained using an animal from the mammal holdings of Lincoln Park Zoological Gardens (Chicago) and I am grateful to the staff of that institution for providing access to that animal. Further cinematographic and all radiographic investigations used animals from a colony I maintained at The University of Michigan. A number of friends assisted with 
the maintenance of this colony and with filming.

Cineradiographic records were obtained using radiographic equipment at the Department of Oral Anatomy at the University of Illinois. Dr. Sue Herring arranged access to those facilities and Mr. R. Graczyk explained and demonstrated the use of the radiographic equipment. Others at the University of Illinois assisted with film and video recording.

This study formed part of a dissertation submitted to the Horace H. Rackham School of Graduate Studies at The University of Michigan. The advice and support of Carl Gans and the other members of the dissertation committee throughout these studies is gratefully acknowledged. Financial support for parts of the present work was provided by the Division of Biological Sciences of The University of Michigan and by a Dr. SchollResearch Fellowship at Lincoln Park Zoo.

\section{LITERATURE CITED}

Auffenberg, W. (1981) The Behavioral Ecology of the Komodo Monitor. Gainesville: University Presses of Florida.

Carlson, H., J. Halbertsma, and M. Zomlefer (1979) Control of the trunk during walking in the cat. Acta Physiol. Scand. 105:251-253.

Daan, S., and T. Belterman (1968) Lateral bending in locomotion of some lower tetrapods. Proc. Kon. Ned. Akad. Wet. (C) 71:245-266.

Edwards, J.L. (1976) The evolution of terrestrial locomotion. In M.K. Hecht, P.C. Goode, and B.M. Hecht (eds): Major Patterns in Vertebrate Evolution. New York: Plenum Press, pp. 553-557.

Gambaryan, P.P. (1974) How Mammals Run: Anatomical Adaptations. New York: John Wiley \& Sons.

Goslow, G.E., Jr., R.M. Reinking, and D.G. Stuart (1973) The cat step cycle: Hind limb joint angles and muscle length during unrestrained locomotion. J. Morphol. 141:1-42.

Hildebrand, M. (1966) Analysis of the symmetrical gaits of tetrapods. Folia Biotheor. 6:9-22.
Hildebrand, M. (1976) Analysis of tetrapod gaits: general considerations and symmetrical gaits. In R.M. Herman, S. Grillner, P.S.G. Stein, and D.G. Stuart (eds): Neural Control of Locomotion. New York: Plenum Publishing Corporation, pp. 203-236.

Howell, A.B. (1944) Speed in Animals. Chicago: University of Chicago Press.

Inman, V.T., H.J. Ralston, and F. Todd (1981) Human Walking. Baltimore: Williams \& Wilkins.

Jenkins, F.A., Jr. (1971) Limb posture and locomotion in the Virginia opossum (Didelphis marsupialis) and other non-cursorial mammals. J. Zool. (Lond.) 165:303-315.

Jenkins, F.A., Jr., and S.M. Camazine (1977) Hip structure and locomotion in ambulatory and cursorial carnivores. J. Zool. (Lond.) 181:351-370.

Kemp, T.S. (1982) Mammal-like Reptiles and the Origin of Mammals. London: Academic Press.

Levens, A.S., V.T. Inman, and J.A. Blosser (1948) Transverse rotation of the segments of the lower extremity in locomotion. J. Bone Joint Surg. [A] 30:859-872.

Pridmore, P.A. (1983) Vertebral bending in locomoting mammals. Am. Zool. 23:889 (abstract).

Rockwell, H., F.G. Evans, and H.C. Pheasant (1938) The comparative morphology of the vertebrate spinal column. Its form as related to function. J. Morphol. 63:86117.

Roos, P.J. (1964) Lateral bending in newt locomotion. Proc. Kon. Ned. Akad. Wet. (C) 67:223-232.

Slijper, E.J. (1946) Comparative biologic-anatomical investigations on the vertebral column and spinal musculature of mammals. Verh. K. Ned. Akad. Wet. (Tweede sectie) 42(5):1-128.

Snyder, R.C. (1962) Adaptations for bipedal locomotion of lizards. Am. Zool. 2:191-203.

Sukhanov, V.B. (1974) General System of Symmetrical Locomotion of Terrestrial Vertebrates and Some Features of Movement of Lower Tetrapods. Washington, DC: Smithsonian Institution and National Science Foundation.

Washburn, S.L., and J. Buettner-Janusch (1952) The definitions of thoracic and lumbar vertebrae. Am. J. Phys. Anthropol. 10:251-252.

Webb, G.J.W., and C. Gans (1982) Galloping in Crocodylus johnstoni-a reflection of terrestrial activity? Rec. Aust. Mus. 34:607-618.

Zug, G.R. (1974) Crocodilian galloping: an unique gait for reptiles. Copeia 1974:550-552. 